\title{
STIMA DELL'EVAPOTRASPIRAZIONE EFFETTIVA MEDIANTE TELERILEVAMENTO AEREO IPERSPETTRALE
}

\author{
Giuseppe Ciraolo, Mario Minacapilli, Maurizio Sciortino
}

\section{INTRODUZIONE}

\section{Introduction}

La quantificazione dei flussi evapotraspirativi da colture irrigue riveste un'importanza fondamentale, in particolare nelle zone semi-aride del Mediterraneo, per la corretta stima dei fabbisogni idrici e, conseguentemente, per l'individuazione di nuove strategie gestionali in grado di aumentare l'efficienza nei sistemi irrigui collettivi. In tal senso, l'esigenza di rendere realmente applicabili nuove procedure in grado di stimare i consumi idrici delle colture su ampie porzioni di territorio, ha indirizzato parte della ricerca scientifica verso lo sviluppo e la messa a punto di modelli matematici in grado di fornire una dettagliata descrizione dei processi di scambio di massa e di energia nel sistema Suolo-Pianta-Atmosfera (SPA), anche attraverso l'utilizzo integrato di misure remote ricavabili da dati telerilevati da piattaforme aeree e/o satellitari. Numerosi studi [2], [15], [19], [21], [26], [28], [30], hanno infatti dimostrato che le tecniche remote rappresentano l'unica via a basso costo che può essere impiegata per determinare la variabilità spazio-temporale delle numerose variabili fisiche necessarie anche nelle applicazioni dei modelli più semplificati. In particolare la modellistica matematica a dati spazialmente distribuiti, basata sull'applicazione del bilancio idrologico nel sistema SPA, può beneficiare delle tecniche di telerilevamento come input, per la stima, mediante dati operanti nelle regioni dello spettro elettromagnetico tra il visibile e l'infrarosso vicino (VIS/ NIR), delle proprietà biofisiche del manto vegetale (indice di area fogliare $L A I$, albedo $\alpha$, altezza della coltura $h_{c}$, ecc.) [15]. Per contro, gli approcci basati sull'applicazione di un modello di bilancio energetico

Memoria presentata il 15.05.2006; accettata il 26.03.2007

G. CIRAOLO, Ricercatore, Dip. di Ingegneria Idraulica ed Applicazioni Ambientali, Università di Palermo.

M. MinaCAPILLI, Funzionario Tecnico, Dip. di Ingegneria e Tecnologie Agro-Forestali, Università di Palermo.

M. SCIORTINO, Ricercatore, ENEA, Roma. di superficie possono utilizzare, oltre ai dati acquisiti nelle regioni del VIS/NIR, quelli derivanti da registrazioni nelle regioni dell'infrarosso termico (TIR) per la stima diretta del termine evapotraspirativo a partire dalla quantificazione dei flussi di calore latente che si manifestano al contorno superiore del sistema SPA.

Oltre agli sforzi di tipo modellistico, nell'ultimo decennio si è registrato anche un rinnovato impulso verso le tecniche di misura locale di pieno campo dei flussi di evapotraspirazione. Grazie alla disponibilità di nuovi sensori micrometeorologici sono state messe a punto nuove tipologie di strumentazioni alternative alla classica e dispendiosa tecnica lisimetrica. In particolare le strumentazioni più recenti (Bowen Ratio, Eddy Correlation, Scintillometri, ecc.) si basano su misure di gradienti e/o fluttuazioni dei termini della diffusione turbolenta (temperatura, umidità, concentrazioni di vapore, flusso di calore sensibile, ecc.) direttamente correlate al flusso di evapotraspirazione [8], [9]. Le suddette tecniche di misura richiedono una preventiva analisi delle caratteristiche di stazionarietà spaziale dei movimenti atmosferici (ventosità e fetch) nonché dell'omogeneità ed estensione della canopy che caratterizza il sito di misura, al fine di determinare, con precisione, l'area di interesse a cui associare il dato misurato.

Nella presente memoria vengono presentati i risultati di un'indagine condotta con l'obiettivo di verificare le potenzialità applicative di una procedura di stima dell'evapotraspirazione effettiva basata sulla risoluzione di un modello di bilancio energetico di superficie (SEBAL - Surface Energy Balance Algorithm for Land) [6], [7]. Il modello SEBAL, utilizzato nello svolgimento dell'indagine, è stato validato localmente (su un'apposita area test sperimentale) utilizzando misure dirette di evapotraspirazione ottenute mediante la tecnica scintillometrica [17], [24].

In seguito l'applicabilità della procedura è stata verificata a scala comprensoriale utilizzando dati iperspettrali registrati dal sensore MIVIS (Multispectral Infrared and Visible Imaging Spectrometer) su piattaforma aerea. 


\section{IL MODELLO SEBAL} (Surface Energy Balance Algorithm for Land)

\section{The SEBAL Model}

Il modello SEBAL, proposto originariamente da Bastiaanssen et al. [6], [7] consente la stima dell'evapotraspirazione effettiva da una superficie vegetata ricorrendo alla ben nota equazione del bilancio energetico scritta nella seguente forma:

$$
\lambda E T=R_{n}-G_{0}-H
$$

in cui $\lambda E T\left[\mathrm{~W} / \mathrm{m}^{2}\right]$ è il flusso di calore latente dovuto al processo evapotraspirativo, $R_{n}\left[\mathrm{~W} / \mathrm{m}^{2}\right]$ è la radiazione netta incidente, $G_{0}\left[\mathrm{~W} / \mathrm{m}^{2}\right]$ è il flusso energetico impiegato nello scambio di calore nel suolo mentre $H$ $\left[\mathrm{W} / \mathrm{m}^{2}\right]$ esprime il flusso energetico di calore sensibile, ossia lo scambio di calore tra la superficie e l'atmosfera generato dal gradiente di temperatura.

Il set di dati in ingresso necessari per l'applicazione del modello SEBAL è costituito da:

1. dati meteo, quali la radiazione solare incidente, l'umidità e la temperatura dell'aria, la velocità del vento;

2. parametri biofisici e radiometrici della vegetazione (albedo, altezza coltura, $L A I$ );

3. grandezze energetiche di superficie quali l'emissività e la temperatura.

Nella schematizzazione originaria, gli autori del modello SEBAL hanno proposto una serie di algoritmi per il calcolo dei termini che compaiono nella (1), facilmente applicabili utilizzando dati telerilevati nelle bande VIS/NIR e TIR ed un numero ridotto di informazioni a terra. A tal riguardo la letteratura scientifica offre numerosi esempi di applicazioni del modello SEBAL su ampie porzioni di territorio in cui si fa ricorso a immagini acquisite da svariate tipologie di sensori aerei e/o satellitari che ne consentono l'applicazione spazialmente distribuita [5], [10].

Nei paragrafi seguenti vengono descritti gli algoritmi per la stima dei flussi energetici che compaiono nella (1) unitamente alla metodologia suggerita per la trasformazione del flusso energetico istantaneo $\lambda E T$ $\left[\mathrm{W} / \mathrm{m}^{2}\right]$ in evapotrapirazione giornaliera $E T_{24}[\mathrm{~mm} / \mathrm{d}]$.

\subsection{CALCOlo DELla RADiAZIONE NETTA}

\subsection{Net radiation estimation}

La radiazione netta $R_{n}$, ossia l'aliquota disponibile alla superficie al netto delle perdite dovute alla riflessione, è il risultato complessivo del bilancio radiativo di una superficie, considerando sia la componente a onde corte (lunghezze d'onda tra 0,3 e 2,5 $\mu \mathrm{m}$ ) sia quelle a onde lunghe (lunghezze d'onda tra 3 e 100 $\mu \mathrm{m})$. Pertanto tale grandezza può essere espressa dalla seguente relazione:

$$
R_{n}=\left(R_{s e}-R_{s u}\right)+\left(R_{l e}-R_{l u}\right)
$$

in cui $R_{s e}$ e $R_{s u}$ rappresentano rispettivamente la densità di flusso radiativo ad onde corte entrante ed uscente mentre $R_{l e}$ e $R_{l u}$ indicano gli analoghi flussi ad onde lunghe. Indicando con $\alpha$ l'albedo di superficie e ricorrendo alla legge di Stefan-Boltzmann per il calcolo $R_{l e}$ e $R_{l u}$, la (2), può essere riscritta nella seguente forma:

$$
R_{n}=(1-\alpha) R_{s e}+\varepsilon_{0}\left(\varepsilon^{\prime} \sigma T_{a}^{4}-\sigma T_{0}^{4}\right)
$$

in cui $\varepsilon^{\prime}$ indica l'emissività apparente dell'atmosfera $[-], \varepsilon_{0}$ l'emissività della superficie $[-], \sigma\left[\mathrm{W} / \mathrm{m}^{2} \mathrm{~K}^{4}\right]$ la costante di Stefan-Boltzmann, mentre $T_{a}$ e $T_{0}$ rappresentano rispettivamente la temperatura dell'aria e della superficie in ${ }^{\circ} \mathrm{K}$.

La stima di $R_{s e}$, in mancanza di misurazioni dirette, può essere agevolmente condotta applicando la formulazione astronomica ampiamente descrittA nelle pubblicazioni FAO [1], e tenendo conto dell'influenza atmosferica mediante la stima della trasmissività $\tau$ dell'atmosfera [Brutsaert, 1982]. Per quanto riguarda il calcolo dei termini $\alpha$ (albedo), $\varepsilon_{0}$ (emissività della superficie), e $T_{0}$ il modello SEBAL propone l'utilizzo di dati telerilevati [32]. In particolare, utilizzando i valori di riflettanza superficiale deducibili da osservazioni remote nelle bande VIS/NIR, la stima dell'albedo $\alpha$, meglio definito come "riflettanza emisferica superficiale spettralmente integrata", può essere facilmente condotta utilizzando algoritmi standard [18], [32] che si basano sull'assunzione di comportamento lambertiano della radiazione riflessa dalla superficie.

SEBAL calcola l'emissività superficiale $\varepsilon_{0}$ utilizzando la seguente formulazione empirica [6], [7]:

$$
\varepsilon_{0}=1.009+0.047 \ln (N D V I)
$$

in cui $N D V I$ [-] rappresenta un indice radiometrico di vegetazione calcolato dai valori di riflettanza $\rho_{r}$ e $\rho_{i}$ rispettivamente nelle bande del rosso e dell'infrarosso prossimo. La temperatura al suolo $T_{0}$ che compare nella (3) può essere infine calcolata a partire dalla "temperatura di brillanza" $T_{b}\left[{ }^{\circ} \mathrm{K}\right]$ misurata nelle bande dell'infrarosso termico mediante la seguente espressione [32]:

$$
T_{0}=\frac{T_{b}}{\varepsilon_{0}^{0.25}}
$$

\subsection{CALCOLO DEL FLUSSO DI CALORE NEL SUOLO}

\subsection{Soil heat flux estimation}

Il flusso di calore nel suolo, $G_{0}\left[\mathrm{Wm}^{-2}\right]$, è in generale esprimibile dalla seguente equazione [20]:

$$
G_{0}=\lambda_{x} \frac{\partial T_{i}}{\partial z}
$$

in cui $\partial T_{s} / \partial \mathrm{z}\left[\mathrm{Km}^{-1}\right]$ esprime il gradiente verticale di temperatura nel suolo e $\lambda_{s}\left[\mathrm{Wm}^{-1} \mathrm{~K}^{-1}\right]$ la conducibilità termica apparente del suolo.

Numerosi studi [16], [25], [5] hanno dimostrato la 
possibilità di derivare, con sufficiente attendibilità, stime di $G_{0}$ utilizzando relazioni empiriche in cui compaiono i termini $R_{n}$ (radiazioni netta), $\alpha$ (albedo), $N D V I$ (indice radiometrico di vegetazione) e $T_{0}$ (temperatura superficiale). In particolare nel modello SEBAL viene proposta la seguente relazione, ampiamente validata sulla base di misure sperimentali condotte in diversi siti [6]:

$$
G_{0}=R_{N} \frac{T_{0}}{\alpha}\left(0.003 \alpha+0.006 \alpha^{2}\right) \times\left(r-0.98 N D V I^{4}\right)
$$

anche in questo caso, i termini che compaiono nella eq. (7) consentono una agevole applicazione su ampie porzioni di territorio utilizzando dati remoti operanti nelle bande VIS/NIR e TIR.

\subsection{CAlColo DEL FluSSO Di CALORE SENSIBILE}

\subsection{Sensible heat flux estimation}

La stima del flusso di calore sensibile $H$ rappresenta l'aspetto più complesso nella risoluzione del bilancio energetico espresso dall'eq. (1). Seguendo uno schema ormai classico della micrometeorologia [8], con riferimento allo strato limite dell'atmosfera più prossimo alla superficie il flusso turbolento di calore sensibile $H\left[\mathrm{Wm}^{-2}\right]$ può essere espresso in funzione del gradiente termico tra la superficie e lo strato più basso dell'atmosfera:

$$
H=\frac{\rho c_{p}}{r_{a t}} \delta T_{a}
$$

in cui $\rho\left[\mathrm{Kg} \mathrm{m}^{-3}\right]$ è la densità dell'aria, $c_{p}$ il calore specifico a pressione costante $\left[\mathrm{JKg}^{-1} \mathrm{~K}^{-1}\right], r_{a h}\left[\mathrm{~s} \mathrm{~m}^{-1}\right]$ la resistenza aerodinamica al trasporto di calore, mentre $\delta T_{a}$ rappresenta la differenza di temperatura $\left(T_{z r e f}\right.$. $T_{z 0 h}$ ) lungo la verticale tra le quote $z_{r e f}$ e $z_{0 h}$, che individuano lo spessore della porzione di strato limite dell'atmosfera considerata per il calcolo del flusso di calore sensibile [8].

Le due quote citate dipendono dalle caratteristiche geometriche e di scabrezza della superficie considerata; nel caso di superfici vegetate numerosi studi [11], [27], [31] hanno dimostrato in genere la validità delle seguenti relazioni empiriche:

$$
\begin{aligned}
z_{\text {ref }} & =z_{\text {inst }}-d \\
z_{\text {oh }} & =0.1 z_{\text {om }}
\end{aligned}
$$

in cui $z_{\text {inst }}[\mathrm{m}]$ indica la quota di misurazione della velocità del vento (in genere $2 \mathrm{o} 10 \mathrm{~m}), d[\mathrm{~m}]$, indica la quota in cui si manifesta la massima resistenza aerodinamica, mentre $z_{o m}$ e $z_{o h}$ indicano, rispettivamente, la lunghezza d'attrito per il trasporto della quantità di moto e la lunghezza d'attrito per lo scambio di calore. In genere, per un ampio campo di colture, $i$ suddetti parametri sono esprimibili in funzione dell'altezza $h_{c}$ della coltura $[\mathrm{m}]$ :

$$
\begin{aligned}
d & =0.63 h_{c} \\
z_{\text {om }} & =0.123 h_{c}
\end{aligned}
$$

La resistenza aerodinamica $r_{a h}\left(\mathrm{sm}^{-1}\right)$ che compare nella (8) è invece esprimibile con la seguente relazione [9], [31]:

$$
r_{a h}=\frac{l}{K u^{*}}\left[\ln \left(\frac{z_{r e f}}{z_{o h}}\right)-\Psi_{h}\right]
$$

in cui $K$ [-] è la costante di von Karman, $u^{*}$ la velocità di attrito $\left[\mathrm{ms}^{-1}\right]$ mentre $\Psi_{h}[-]$ rappresenta un coefficiente di correzione della stabilità atmosferica [31] trascurabile solo in condizioni di neutralità atmosferica (gradiente della temperatura potenziale adiabatico).

Nella (13) la determinazione della velocità di attrito $u^{*}$ può essere condotta, nell'ipotesi di distribuzione verticale della velocità di tipo logaritmo, utilizzando la seguente espressione [9]:

$$
u^{*}=\frac{K u_{200}}{\ln \left(\frac{200-d}{z_{\text {ow }}}\right)-\Psi_{m}}
$$

in cui $\mathrm{u}_{200}\left[\mathrm{~ms}^{-1}\right]$ è la velocità del vento misurata o dedotta ad un'altezza di 200 metri e $\Psi_{m}$ [-] rappresenta un ulteriore coefficiente di correzione della stabilità atmosferica [31]. Per le superfici naturali e nelle applicazioni a ridotta scala temporale (giorno o ora) l'ipotesi di neutralità è difficilmente applicabile, pertanto, in questi casi, la corretta applicazione delle eq. (8), (13) e (14) non può prescindere dalla stima di $\Psi_{h} \mathrm{e} \Psi_{m}$.

Tralasciando, per brevità, la descrizione delle formulazioni che consentono il calcolo di $\Psi_{h}$ e $\Psi_{m}$ [31] occorre però sottolineare che esse dipendono da $\mathrm{H} \mathrm{e}$ quindi da $r_{a h}$. Pertanto la risoluzione del sistema di eq. (8), (13) e (14) viene in genere conseguita per via iterativa, in cui, al primo passo, si ipotizzano condizioni neutrali. Successivamente, partendo da un primo valore $r_{a h}$ calcolato tramite la (13) con $\Psi_{h}=0$, si deducono dei valori di primo tentativo sia di $H$ (eq. 8) che di $\mathrm{u}^{*}$; da essi è possibile ricalcolare $r_{a h}$ tramite la eq.(13) e quindi, per successive iterazioni (in genere < 10), ottenere un valore finale di $H$ che soddisfa opportuni vincoli di convergenza numerica. In SEBAL viene proposto l'utilizzo delle eq. (8), (13) e (14) unitamente alla tecnica iterativa per la stima di $H$.

Per quanto riguarda la stima della differenza di temperatura $\delta T_{a}$ che compare nell'eq. (8) occore innanzitutto precisare che l'utilizzo di dati TIR da osservazioni remote non ne consente una stima diretta a causa della non corrispondenza tra la temperatura radiometrica e quella aerodinamica. A tal fine SEBAL propone una procedura indiretta per la stima distribuita del termine $\delta T_{a}$ che compare nella (8), in funzione della temperatura superficiale $T_{0, i}$ di ciascun pixel dell'immagine. A tal fine, ipotizzando l'esistenza di una relazione di tipo lineare tra $\delta T_{a, i}$ e $T_{0, i}$ (dove il pedice $i$ indica il generico pixel), il modello SEBAL propone l'utilizzo della seguente relazione:

$$
\delta T_{a, i}=b T_{0, i}+a
$$

in cui $b$ ed $a$ sono due coefficienti di calibrazione deducibili definendo due opportune condizioni limite 
per le quali risulta possibile, dall'eq. (8), ricavare $\delta T_{a,}$. Nel SEBAL le condizioni limite vengono individuate in corrispondenza di due opportuni pixel dell'immagine denominati "pixel freddo" e "pixel caldo" di cui è noto rispettivamente il valore di $T_{0, i}$. In corrispondenza del "pixel freddo", individuabile sull'immagine in corrispondenza, ad esempio, di superfici densamente vegetate in cui è lecito ipotizzare che la temperatura superficiale sia prossima a quella dell'aria, la stima del calore sensibile può essere agevolmente condotta utilizzando l'equazione di bilancio energetico (eq.1) in cui i termini $R_{n}$ e $G_{0}$ vengono direttamente calcolati utilizzando le eq. (3) e (7), ed ipotizzando un flusso evapotraspirativo massimo calcolabile con la nota formulazione di Penman-Monteith [1]. In maniera analoga, in corrispondenza del "pixel caldo", individuabile sull'immagine in corrispondenza di suoli nudi abbastanza secchi, la stima di $H$ può essere dedotta considerando nullo il termine evapotraspirativo. Noti i valori di $T_{0}, H$ e di $r_{a h}$ in corrispondenza dei suddetti pixel, è possibile per entrambi calcolare i relativi valori di $\delta T_{a}$ che, di conseguenza consentono la stima, tramite la risoluzione di un sistema di due equazioni (eq.15 applicata al "pixel caldo" e al "pixel freddo"), nelle due incognite $a$ e $b$.

\subsection{CALCOLO DELL'EVAPOTRASPIRAZIONE GIORNALIERA}

\subsection{Daily evapotranspiration estimation}

Noti i termini $R_{n}, G_{0}$ ed $H$ l'eq. (1) consente il calcolo di $\lambda E T$ espresso come flusso radiativo istantaneo $\left[\mathrm{Wm}^{-2}\right]$. Nel modello SEBAL il passaggio dal valore istantaneo al valore giornaliero di $\lambda E T$, e quindi la stima dell'evapotraspirazione $E T_{24}$ espressa in $\mathrm{mmd}^{-1}$, viene condotta utilizzando la "frazione evaporativa" $\Lambda$, pari al rapporto adimensionale tra il flusso di calore latente $\lambda E T$ ed il flusso di energia disponibile in superficie $\left(R_{n}-G_{0}\right)$ [27]:

$$
\Lambda=\frac{\lambda E T}{R_{s}-G_{0}}
$$

Diversi studi [10], [13] hanno dimostrato che nel corso delle ore diurne della giornata, i valori di $\Lambda$ [01] sono pressochè costanti nel tempo. $\Lambda$ è stato dunque utilizzato come parametro di integrazione temporale per il calcolo dei valori di evapotraspirazione giornaliera $\left[\mathrm{mm} \mathrm{d}^{-1}\right]$ tramite la seguente espressione:

$$
E T_{24}=\Lambda \frac{R_{9.24}}{\lambda}
$$

in cui $\lambda\left[\mathrm{MJKg}^{-1}\right]$ è il calore latente di vaporizzazione ed $R_{n, 24}$ rappresenta la radiazione netta giornaliera che può essere facilmente dedotta utilizzando le note formulazioni proposte nelle pubblicazioni FAO [1].

\section{DESCRIZIONE DELL'AREA DI STUDIO E DELLE INDAGINI EFFETTUATE}

\section{Case study and applications}

L'area selezionata per lo studio (figura 1) ricade nel comprensorio irriguo siciliano "Lago Arancio" e si estende per circa 850 ettari tra le provincie di Agrigento, Trapani e Palermo.

Le colture più diffuse sono di tipo arboreo con prevalenza di olivo, vite ed agrumi. Il comprensorio è servito da una rete in pressione di recente realizzazione, dotata di apparecchiature di telecomando e di telecontrollo che consentono la registrazione dei volumi consegnati alle singole utenze. La modalità di consegna dei volumi irrigui è del tipo cosiddetto "a domanda programmata".

Dal punto di vista pedologico, nella zona occidentale dell'area considerata prevalgono i terreni pianeggianti caratterizzati da associazioni di suoli bruni a tessitura media o medio-sabbiosa, mentre nella zona orientale i terreni sono caratterizzati da una morfologia leggermente ondulata con prevalenza di vertisuoli di tessitura medio-limoso-argillosa [14].

All'interno dell'area di studio (figura 1) sono state selezionate due diverse sottozone utilizzate per la validazione a scala di campo (sottozona A) e per l'applicazione distribuita a scala sub-comprensoriale (sottozona B) della procedura SEBAL.

Per le suddette sottozone due intense campagne sperimentali di acquisizione sono state condotte nel 2002 - periodo nel quale nella sottozona B è stato programmato un volo aereo per l'acquisizione dei dati iperspettrali MIVIS - e nel 2005 - in cui sono stati raccolti, all'interno di un'azienda ricadente nella sottozona A, una serie di misure acquisite da una stazione agroclimatica e da uno scintillometro per la misure dei flussi energetici su un appezzamento di terreno coltivato ad erba medica.

Durante la stagione irrigua 2002 l'area irrigua ricadente nella sottozona $\mathrm{B}$ è stata ripresa dal sensore iperspettrale aviotrasportato MIVIS (Multispectral Infrared and Visible Imaging Spectrometer) che ha sorvolato la zona alle ore 14.00 del 19/06/2002 ad una quota di circa 1500 metri. L'intera area è stata acquisita con 4 immagini adiacenti caratterizzate da una risoluzione a terra di circa 3 metri.

Da un punto di vista radiometrico (Tabella 1) nelle bande VIS/NIR (1-92) il dato MIVIS fornisce una misura di radianza al sensore espressa in $\mathrm{W} / \mathrm{cm}^{2} / \mathrm{sr} / \mu \mathrm{m}$, per le bande TIR (93-102) il dato MIVIS è invece una temperatura espressa in gradi Celsius.

Contemporaneamente al volo MIVIS è stata condotta una campagna di misure di campo su target a terra localizzati in corrispondenza di 20 campi coltivati a vite, olivo ed agrumeto. Per ciascuno di essi è stato misurato il valore medio dell'indice di area fogliare LAI mediante l'utilizzo della strumentazione portatile LAI2000 della Licor [22].

In corrispondenza di altri 5 target, utilizzando uno 


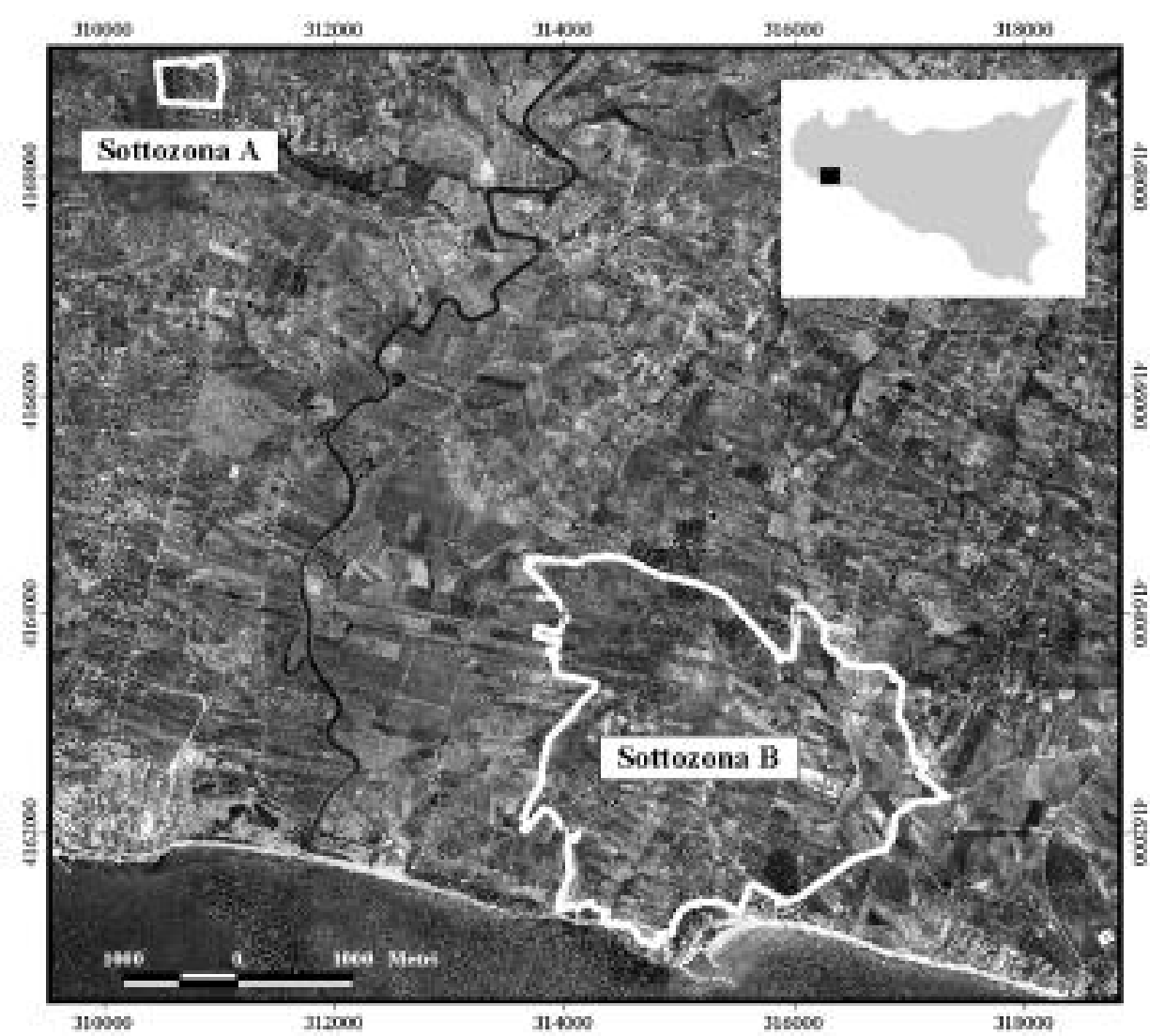

Fig. 1 - Localizzazione geografica dell'area di studio.

Fig. 1 - Geographic location of study area.

spettroradiometro portatile da campo, è stata invece condotta una campagna spettro-radiometrica finalizzata all'acquisizione di misure di riferimento per la correzione radiometrica delle 4 immagini. A tal fine $\mathrm{i}$ suddetti target di riferimento sono stati localizzati in corrispondenza di superfici omogenee spettralmente differenti (asfalto, sabbia, suolo nudo, vegetazione).

Dell'intera informazione radiometrica contenuta nei dati MIVIS sono state utilizzate le prime 20 bande ricadenti nell'intervallo spettrale VIS/NIR compreso nel range 450-830 nm, e due bande TIR (100 e 101) comprese nel range $8200-12700 \mathrm{~nm}$.

Le prime 20 bande VIS/NIR sono state calibrate in riflettanza superficiale mediante una trasformazione empirico-lineare dedotta sulla base delle firme acquisite in corrispondenza dei 5 target di calibrazione [32].

Le due bande termiche non hanno invece richiesto una preventiva calibrazione poiché $\mathrm{i}$ valori di temperatura superficiale registrati sono risultati in accordo con i valori misurati in campo contemporaneamente all'acquisizione.

Successivamente alla fase di calibrazione radiometrica l'intero data-set è stato corretto geometricamente utilizzando la tecnica "Rubber-Sheeting", implementata nel pacchetto software Erdas-Imagine [18]. A tal fine, sono stati localizzati circa 250 punti di controllo in ciascuna immagine grazie all'ausilio di una ortofo- to digitale della stessa zona. Tale elaborazione ha consentito la corretta mosaicatura delle 4 immagini adiacenti e la georeferenzazione in un unico sistema di riferimento.

In figura 2 vengono rappresentati i risultati delle suddette elaborazioni: in particolare la figura 2.a mostra una composizione di bande del visibile, mentre in figura 2.b viene rappresentata la mappa di temperatura superficiale dedotta dalla banda TIR $n^{\circ} 100$.

Durante la stagione irrigua 2005 un'intensa attività sperimentale è stata condotta all'interno della sottozona $A$, al fine di acquisire un data-base di misure di flussi energetici e di parametri biofisici e colturali da utilizzare per la validazione del modello SEBAL a scala di campo. In particolare all'interno di un'azienda agricola sperimentale gestita dal Servizio Informativo Agrometeorologico Siciliano (SIAS) è stato predisposto un campo coltivato ad erba medica di estensione par a circa 0.35 ha in cui è stato collocato uno scintillometro laser per la misura dei flussi energetici $\left(R_{n}, G_{0}, H\right)$.

Per lo svolgimento dell'indagine sono stati utilizzati anche i dati acquisiti da una stazione agroclimatica del servizio SIAS collocata all'interno dell'azienda e in grado di registrare, a scala oraria, i valori di temperatura dell'aria, del suolo, ventosità, umidità dell'aria, radiazione solare e precipitazione. Lo scintillometro utilizzato (modello Scintec SLS20) è costituito da 


\begin{tabular}{|c|c|c|}
\hline Bande & Interv. Spett. [nm] & Ampiezza [nm] \\
\hline $1-20$ & $430-830$ & 20 \\
\hline $21-28$ & $1150-1550$ & 50 \\
\hline $29-92$ & $2000-2500$ & 9 \\
\hline $93-102$ & $8200-12700$ & $34-54$ \\
\hline
\end{tabular}

TABELla 1 - Caratteristiche del sensore MIVIS.

TABLE 1 - Mivis sensor characteristics.

una stazione agroclimatica dotata di sensori per la misura della radiazione netta e globale, della pressione atmosferica, dei gradienti di temperatura e dei flussi di calore nel suolo; la misura dei flussi di calore sensibile viene invece derivata dalle fluttuazioni dell'intensità turbolenta dell'indice di rifrazione dell'aria registrate da un ricevitore laser in grado di misurare le vibrazioni di un fascio di luce emesso da un apposito trasmettitore [24]. La figura 3 schematizza la componente sensoristica dello scintillometro utilizzato; i dati registrati con un intervallo minimo di 2 minuti sono stati elaborati dal software di acquisizione che consente la registrazione di tutti i termini del bilancio energetico e l'integrazione a scala oraria e/o giornaliera. I suddetti valori consentono infine di ricavare, come termine residuo del bilancio radiativo, i flussi di evapotraspirazione dall'area di influenza dello scintillometro la cui configurazione varia in funzione della geometria di installazione. Nel caso in studio, al fine di ottenere misure riferite al solo campo di erba medi$\mathrm{ca}$, il trasmettitore ed il ricevitore dello scintillometro sono stati collocati ad una distanza di circa 80 metri lungo la direzione maggiore del campo e ad una altezza di circa $70 \mathrm{~cm}$ al disopra della superficie vegetata.

Le registrazioni, acquisite dal 16/05/2005 fino alla fine del mese di agosto, sono state aggregate ed analizzate a scala oraria; durante questo periodo il campo

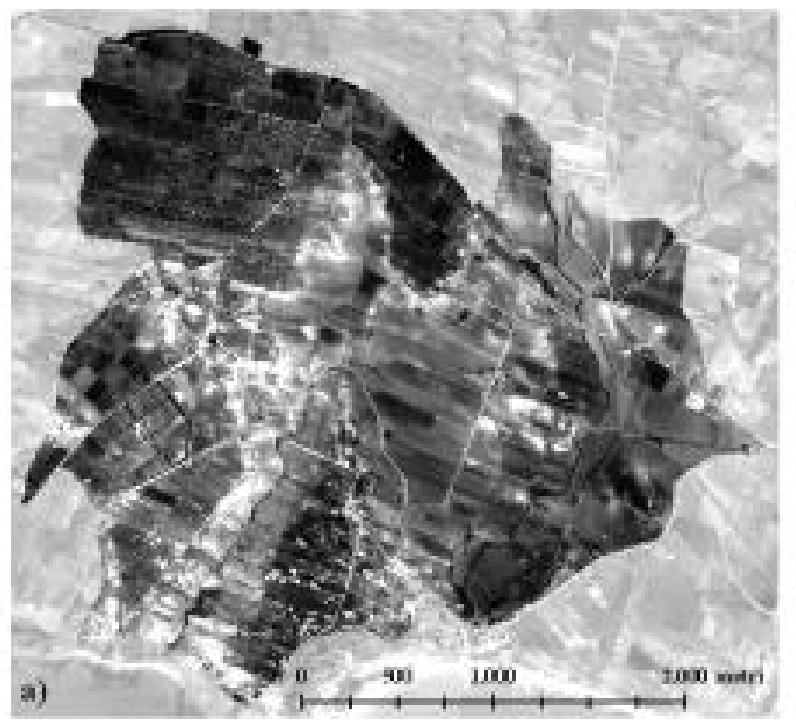

di erba medica è stato periodicamente irrigato e sfalciato mantenendo regimi di stress controllato ed un'altezza colturale inferiore ai $50 \mathrm{~cm}$.

\section{RISULTATI E DISCUSSIONE}

\section{Results and discussion}

\subsection{VALIDAZIONE A SCALA DI CAMPO}

\subsection{Validation at field scale}

Durante la stagione irrigua 2005 i dati acquisiti dall'installazione sperimentale descritta nel paragrafo precedente hanno consentito lo svolgimento di un'analisi di validazione del modello SEBAL sulla base delle misure dei flussi energetici acquisiti dallo scintillometro. Pertanto, al fine di rendere maggiormente significativa l'indagine, sono state utilizzate le registrazioni acquisite nei giorni in cui la coltura utilizzata per la sperimentazione (erba medica) era caratterizzata da una fase di effettivo stress idrico. A tal fine da un primo confronto fra le misure scintillometriche ed i valori di evapotraspirazione di riferimento $\left(\mathrm{ET}_{0}\right)$ ottenuti utilizzando la nota formulazione di PenmanMonteith [1], sono stati prescelti i dati registrati nei giorni 14/07, 15/07 e 20/07 in cui l'evapotraspirazione effettiva misurata dallo scintillometro è risultata significativamente inferiore a quella di riferimento. Per queste date, l'applicazione del modello SEBAL è stata inizialmente condotta a scala oraria; successivamente è stata verificata anche la procedura descritta nel paragrafo 2.4 per il calcolo della frazione evaporativa $\Lambda$ e la successiva stima dell'evapotraspirazione giornaliera. La tabella 2 sintetizza i principali dati di input di tipo radiometrico (temperatura ed albedo) utilizzati per l'applicazione del modello SEBAL sulla

Fig. 2 - a) Immagine MIVIS utilizzata per l'indagine; b) Immagine termica ottenuta dalla banda 100.

Fig. 2 - a) MIVIS image used in the application; b) Surface temperature computed using 100 MIVIS band.

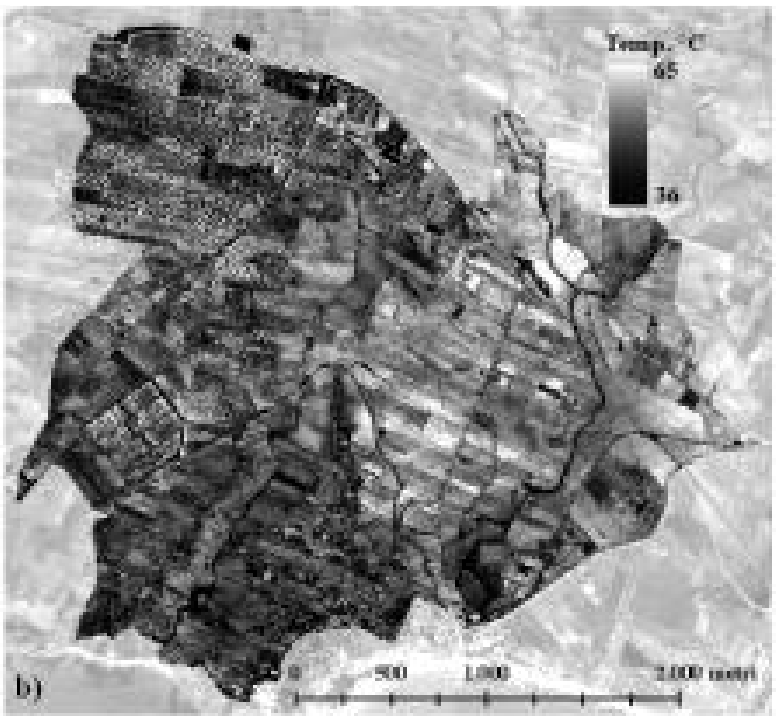


base delle espressioni riportate nei paragrafi 2.1, 2.2, 2.3 e 2.4 ; nella stessa tabella viene riportato anche il valore di frazione evaporativa $\Lambda$ dedotto sulla base delle misure di $R_{n}, G_{0}$ e $\lambda E T$ ottenute dallo scintillometro.

Le informazioni radiometriche di input nelle bande VIS-NIR e TIR sono state rispettivamente dedotte dalle misure spettroradiometriche e termiche acquisite in campo mediante uno spettroradiometro portatile da campo ed un termometro radiometrico. Le misure per la calibrazione dei coefficienti $a$ e $b$ che compaiono nell'eq.(15) sono state condotte su un suolo nudo ed asciutto (pixel caldo) e sulla chioma di un agrumeto irriguo (pixel freddo) caratterizzato da un valore di $L A I$ pari a 3 . I valori dell'indice di vegetazione $N D V I$ che compare nell'eq.(7) sono stati calcolati a partire dai valori di riflettanza nelle bande del rosso e del vicino infrarosso misurati direttamente in campo tramite uno spettroradiometro portatile. Data la modesta variabilità misurata durante le ore di acquisizione (9.0017.00) si è ritenuto opportuno fissare un valore costante di NDVI ottenuto dalla media dei valori misurati.

I grafici riportati nelle figure $4 \mathrm{a}, 4 \mathrm{~b}$ e $4 \mathrm{c}$ mostrano il confronto fra le stime di evapotraspirazione effettiva ottenute dal modello SEBAL, i valori misurati dallo scintillometro durante i tre giorni di sperimentazione, nonchè i valori dell'evapotraspirazione della coltura di riferimento $\mathrm{ET}_{0}$ dedotti con la nota formulazione di Penman-Monteith [1]. Gli andamenti riportati mostrano un sostanziale accordo tra le misure registrate in campo e le simulazioni ottenute dal modello SEBAL, risultate mediamente sovrastimate di un valore pari a circa il 4\%. Le maggiori discrepanze si sono registrate nelle ore estreme dell'intervallo di misurazione (9.00-17.00) dove solo in un caso su 27 è stata registrata una sovrastima dei valori simulati rispetto alle misurazioni pari a circa il $31 \%$; nelle ore centrali

\begin{tabular}{|c|c|c|c|c|c|c|}
\hline & ora & $\begin{array}{c}T_{\text {eold }} \\
{\left[{ }^{\circ} \mathrm{C}\right]}\end{array}$ & $\begin{array}{l}T_{\text {hor }} \\
{\left[{ }^{\circ} \mathrm{C}\right]}\end{array}$ & $\begin{array}{l}T_{s} \\
{[-C]}\end{array}$ & $\begin{array}{l}\alpha \\
{[-]}\end{array}$ & $\begin{array}{l}\bar{C} \\
{[-]}\end{array}$ \\
\hline \multirow{9}{*}{$\begin{array}{l}\text { 总 } \\
\text { d } \\
\text { 点 } \\
\text { 妾 }\end{array}$} & 900 & 26.8 & 53.0 & 26.9 & 0.39 & 0.73 \\
\hline & 10.00 & 28.5 & 56.2 & 20.7 & 0.36 & 0.68 \\
\hline & 11.00 & 29.8 & 59.4 & 32.3 & 0.32 & 0.67 \\
\hline & 12.00 & 30.7 & 62.5 & 34.7 & 0.27 & 0.67 \\
\hline & 13.00 & 31.3 & 63.2 & 35.2 & 0.23 & 0.68 \\
\hline & 14.00 & 31.6 & 63.7 & 35.6 & 0.21 & 0.66 \\
\hline & 15,00 & 31.2 & 61.1 & 33.7 & 0.22 & 0.68 \\
\hline & 16.00 & 30.7 & 59.1 & 32.2 & 0.26 & 0.67 \\
\hline & 17,00 & 29.9 & 56.6 & 30.5 & 0.36 & 0.66 \\
\hline \multirow{9}{*}{ 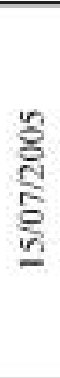 } & 900 & 279 & 54.4 & 28.2 & 0.35 & 0.76 \\
\hline & 10,00 & 29.5 & 57.6 & 30.9 & 0.34 & 0.67 \\
\hline & 11.00 & 31.0 & 61,2 & 33.9 & 0.32 & 0.66 \\
\hline & 12.00 & 31.9 & 64.3 & 36.6 & 0.27 & 0.66 \\
\hline & 13.00 & 32.8 & 65.5 & 37.5 & 0.24 & 0.66 \\
\hline & 14.00 & 33.2 & 66.7 & 38.4 & 0.22 & 0.64 \\
\hline & 15.00 & 33.0 & 63.9 & 35.9 & 0.23 & 0.65 \\
\hline & 16.00 & 32.6 & 62.3 & 34.8 & 0.27 & 0.64 \\
\hline & 17.00 & 31.9 & 60.2 & 33.5 & 0.38 & 0.63 \\
\hline \multirow{9}{*}{$\frac{\mathscr{8}}{\frac{8}{5}}$} & 9.00 & 28.3 & 54.9 & 28.8 & 0.31 & 0.79 \\
\hline & 10,00 & 30.6 & 58.6 & 31.9 & 0.24 & 0.76 \\
\hline & 11.00 & 32.3 & 62.1 & 35.0 & 0.26 & 0.72 \\
\hline & 12.00 & 33.3 & 65.7 & 37.6 & 0.25 & 0.67 \\
\hline & 13,00 & 33.8 & 66.0 & 37.7 & 0.23 & 0.67 \\
\hline & 14,00 & 33.7 & 66.4 & 38.1 & 0.23 & 0.67 \\
\hline & 15.00 & 33.4 & 63.9 & 36.2 & 0.25 & 0.66 \\
\hline & 16,00 & 326 & 61.0 & 34.1 & 0.30 & 0.63 \\
\hline & 17.00 & 31.7 & 58.6 & 32.3 & 0.43 & 0.56 \\
\hline
\end{tabular}

TABella 2 - Parametri misurati e dedotti in campo durante la sperimentazione: $T_{\text {cold }}$ (temp. nel pixel freddo), $T_{\text {hot }}$ (temp. nel pixel caldo), $\alpha$ (albedo), $\Lambda$ (frazione evaporativa).

TABLE 2 - Measured variables obtained during the field investigation: $\mathrm{T}_{\text {cold }}$ (cold-pixel temperature) $\mathrm{T}_{\text {hot }}$ (hot-pixel temperature, $\alpha$ (albedo), $\Lambda$ (evaporative fraction).

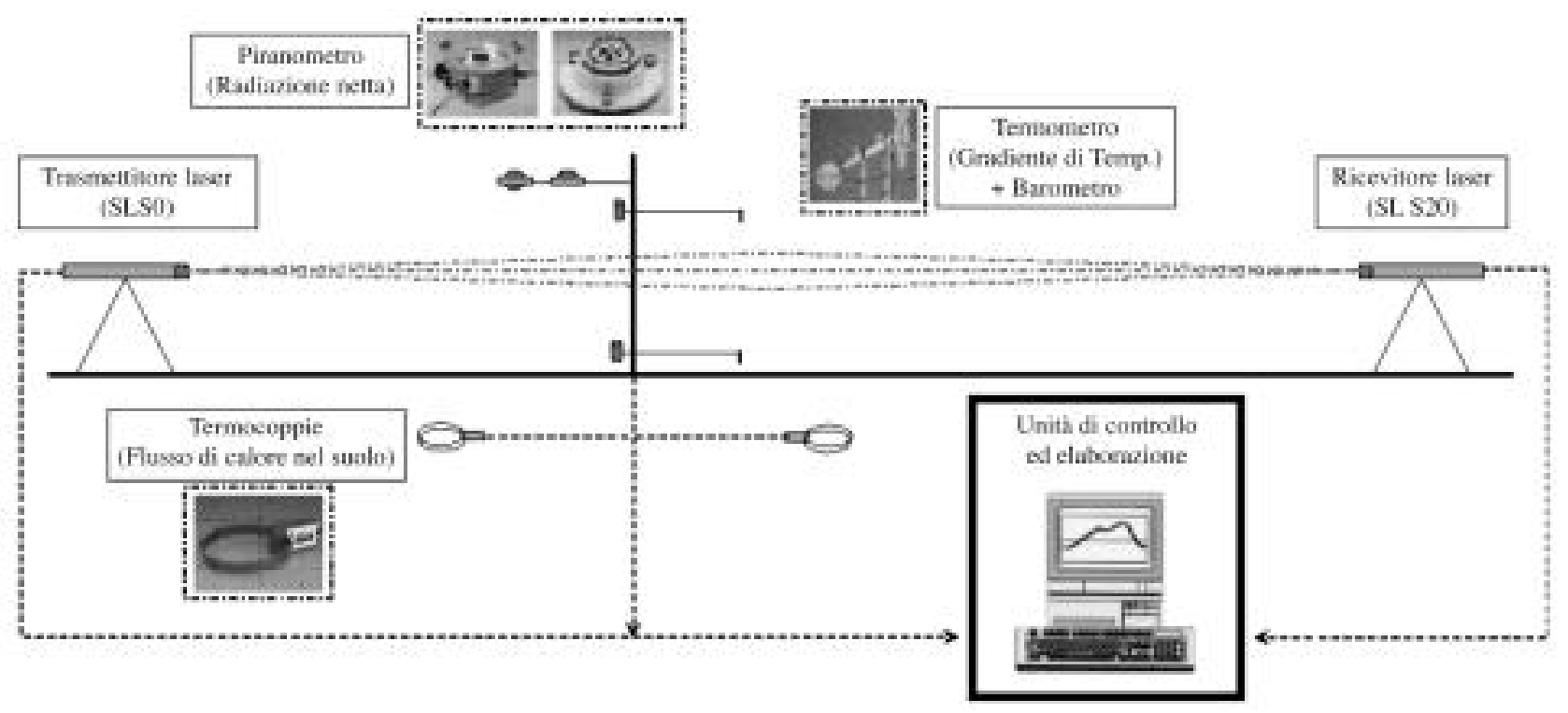

Fig. 3 - Schema della componente sensoristica dello scintillometro utilizzato nell'indagine.

Fig. 3 - General scheme and sensors of the scintillometer used in the study. 

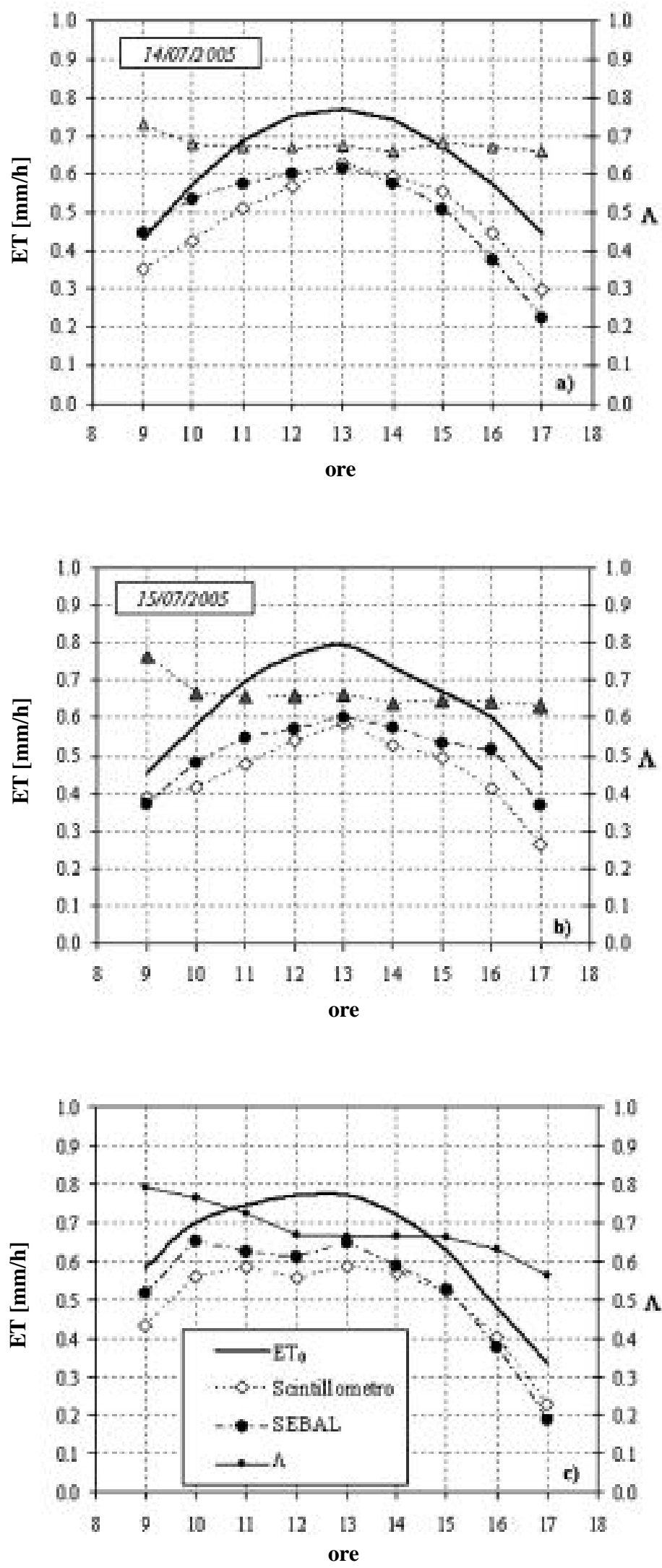

Fig. 4 - Andamenti temporali dei valori di evapotraspirazione oraria misurata (Scintillometro) e simulata con il modello SE$B A L$; nei grafici sono riportati anche $i$ valori di frazione evaporativa $\Lambda$ e di evapotraspirazione di riferimento $\left(E T_{0}\right)$.

Fig. 4 - Temporal variation of measured and simulated hourly evapotranspiration; in the graphs the evaporative fraction $\Lambda$ and $\mathrm{ET}_{0}$ values are plotted. comprese tra le 12.00 e le 14.00 gli scarti sono risultati sempre inferiori al $10 \%$.

Negli stessi grafici è stato riportato anche l'andamento della frazione evaporativa $\Lambda$, calcolata mediante l'eq.(16) ed utilizzando i valori di $\lambda E T, R_{n}$ e $G_{0}$ misurati direttamente dallo scintillometro. Come riscontrato in letteratura [6], [7] il parametro $\Lambda$ è risultato poco variabile soprattutto durante le ore centrali del giorno, avvalorando la possibilità di stimare il flusso evapotraspirativo giornaliero [mm/d] mediante l'eq.(17).

In particolare nel caso in esame i valori medi giornalieri di $\Lambda$ sono risultati compresi tra 0.66 e 0.69 , con una variabilità oraria compresa tra un massimo di 0.79 ed un minimo di 0.63 ; i valori di deviazione standard del dato orario di $\Lambda$ all'interno della giornata sono risultati compresi tra un minimo di 0.021 ed un massimo di 0.038 evidenziando ulteriormente la contenuta variabilità temporale del suddetto parametro.

Quanto sopra evidenziato ha consentito di validare la procedura descritta nel paragrafo 2.4 per la stima dell'evapotraspirazione giornaliera $E T_{24}$ mediante l'eq.(17). A tal fine sono state utilizzate le misure termiche e radiometriche registrate alle ore 12.00 ed evidenziate in tabella 2. Le misurazioni sono state utilizzate per il calcolo di $\Lambda$ tramite l'eq.(16) e per la stima di di $E T_{24}$ tramite l'eq.(17).

Il grafico di figura 5 sintetizza il risultato finale della validazione condotta; in particolare è stato riscontrato un sostanziale accordo tra i valori di $E T_{24}$ ottenuti dal modello SEBAL e gli analoghi valori dedotti dalle misure scintillometriche. Nello stesso grafico sono riportati anche i valori di $\Lambda$ calcolati mediante l'eq.(16) ed utilizzando i valori di $\lambda E T, R_{n}$ e $G_{0}$ ottenuti dal modello SEBAL; anche in questo caso le stime ottenute sono risultate in accordo con i valori misurati riportati nei grafici $4 \mathrm{a}, 4 \mathrm{~b}$ e $4 \mathrm{c}$.

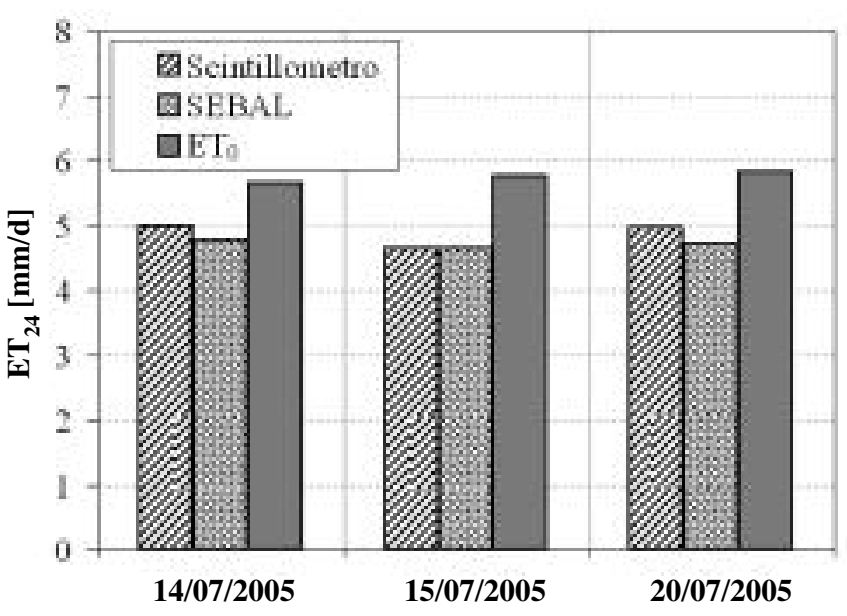

Fig. 5 - Valori di evapotraspirazione giornaliera $E T_{24}$ misurata (Scintillometro) e stimata dal modello SEBAL. Il grafico riporta anche $i$ valori di evapotraspirazione di riferimento $E T_{0}$.

Fig. 5 - Comparison between SEBAL simulated and measured daily evapotranspiration $\mathrm{ET}_{24}$. The graph shows also the $\mathrm{ET}_{0}$ values. 


\subsection{APPLICAZIONE A SCALA COMPRENSORIALE}

\subsection{District scale application}

Come evidenziato nel paragrafo 3 l'applicazione del modello SEBAL a scala comprensoriale è stata condotta nella sottozona B dell'area di studio (Fig. 1) utilizzando le immagini MIVIS acquisite durante il volo del 19/06/2002. A tal fine partendo dai valori di riflettanza superficiale registrati per ciascun pixel dell'immagine (Fig. 2), sono state ricavate le distribuzioni spaziali di albedo $(\alpha)$ e dell'indice di vegetazione $N D V I$ che compare nell'equazione (7). Le stime dell'indice di area fogliare $L A I$ e dell'altezza colturale $h_{c}$ sono state condotte utilizzando un set di relazioni empiriche presenti in letteratura [19], [12] la cui applicabilità nell'area di studio è stata verificata in una precedente indagine [23].

Infine per quanto riguarda l'informazione radiometrica nelle bande del termico, sono stati utilizzati i valori di temperatura superficiale dedotti dalla banda 100 del sensore MIVIS. Queste informazioni hanno consentito di definire tutti gli input di base necessari per l'applicazione spazialmente distribuita degli algoritmi che compaiono nel modello SEBAL e descritti nel paragrafo 2 .

L'implementazione del modello SEBAL è stata effettuata utilizzando il linguaggio di programmazione "Model-Builder Macro language" del software ErdasImagine [18] utilizzato per l'elaborazione delle immagini MIVIS.

Le figure $6 a, b, c$, mostrano, relativamente all'area di studio le distribuzioni spaziali dei valori istantanei di radiazione netta $R_{n}$, flusso di calore nel suolo $G_{0}$ e flusso di calore sensibile $H$ espressi in $\mathrm{W} / \mathrm{m}^{2}$. Tali distribuzioni hanno consentito di ricavare, per ogni pixel dell'immagine, i valori di frazione evaporativa $\Lambda$ necessari per l'applicazione dell'eq.(17)

Il calcolo della radiazione netta giornaliera $R_{n 24}$ è stato invece condotto utilizzando le note formulazioni descritte nelle pubblicazioni FAO [1].

Le grandezze calcolate hanno consentito infine di ricavare, per ogni pixel dell'immagine, i valori di evapotraspirazione effettiva giornaliera, espressa in $\mathrm{mm} / \mathrm{d}$, la cui distribuzione spaziale è rappresentata in figura 7. La mappa mostra valori di $E T_{24}$ variabili nel range compreso tra 0 e $5 \mathrm{~mm} / \mathrm{d}$; i valori maggiori risultano per lo più localizzati in corrispondenza delle colture maggiormente vegetate o in suoli nudi ad elevato tasso di umidità.

L'elevata risoluzione geometrica (3 metri) della distribuzione spaziale dei valori di $E T_{24}$ ha infine consentito di calcolare il valore medio di evapotraspirazione giornaliera all'interno di aree omogenee corrispondenti a 200 parcelle irrigue localizzate nell'area test ed identificate grazie alle tecniche di sovrapposizione geografica fra immagini telerilevate e cartografia numerica. Il grafico di figura 8 sintetizza in particolare il risultato dell'analisi: i valori medi di $E T_{24}$ campionati su 18 agrumeti, 20 oliveti e 141 vigneti sono risultati rispettivamente pari a $2.47 \mathrm{~mm} / \mathrm{d}$,
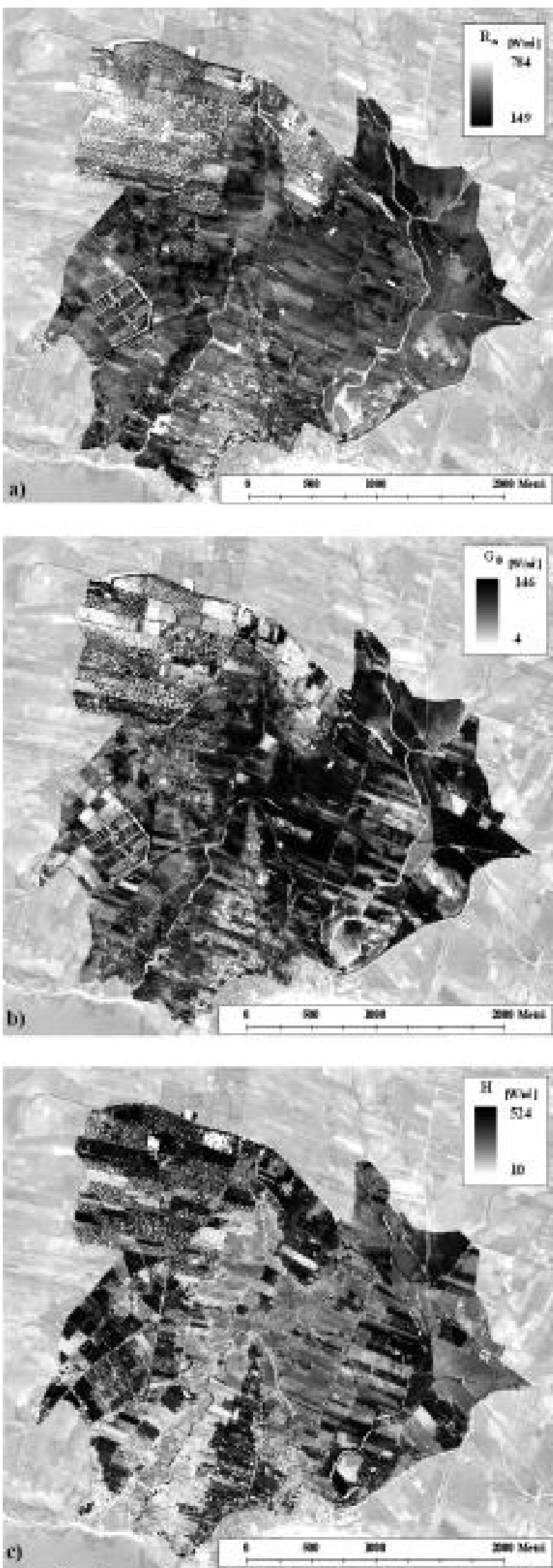

Fig. 6-a,b,c-Mappe dei valori di radiazione netta $R_{n}$, flusso di calore nel suolo $G_{0}$ e flusso di calore sensibile $H$.

Fig. 6-a,b,c-Maps of net radiation $R_{n}$, soil heat flux $G_{0}$ and sensibile heat flux $\mathrm{H}$ 


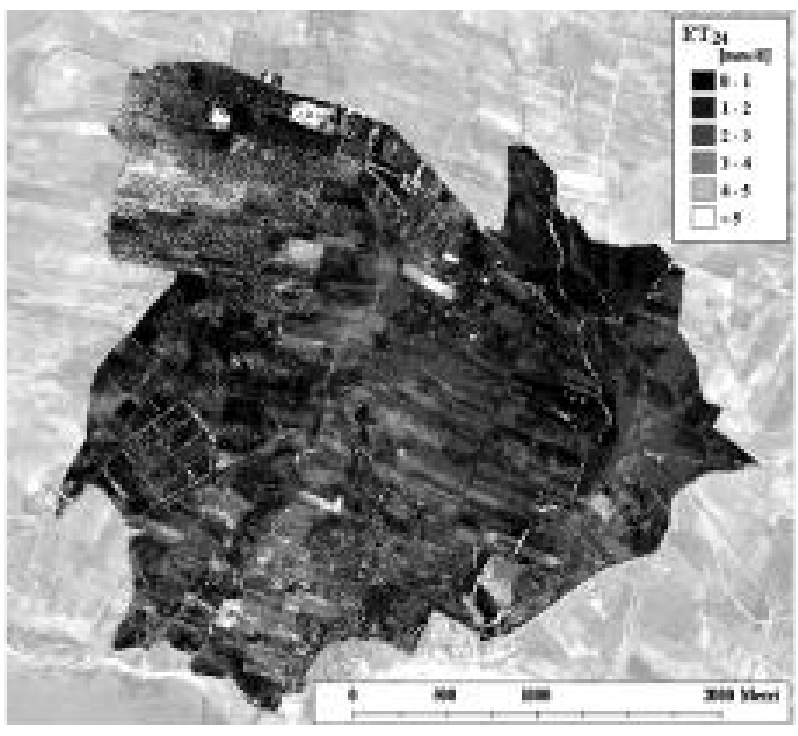

Fig. 7 - Distribuzione spaziale dei valori di evapotraspirazione effettiva giornaliera.

Fig. 7 - Spatial distribution of daily evapotraspi-ration.

$2.32 \mathrm{~mm} / \mathrm{d}$ e $1.78 \mathrm{~mm} / \mathrm{d}$; nello stesso grafico sono riportanti anche i relativi range di variabilità.

Le suddette stime, ed in particolare la possibilità di analizzare la variabilità spaziale del processo evapotraspirativo all'interno di un'area irrigua, rappresentano, in definitiva, il risultato più importante dell'indagine condotta dalla quale è emerso l'importante ruolo delle tecniche di Telerilevamento nella modellazione agro-idrologica a scala territoriale. In particolare appare evidente la possibilità di migliorare la ricaduta operativa derivabile dall'applicazione di tali tecniche con sensori satellitari dotati di adeguata risoluzione spettrale, geometrica e temporale. A tal proposito la letteratura scientifica [21] propone già nuove metodologie utili alla disaggregazione di dati TIR satellitari per la generazione di mappe di temperatura con riso-

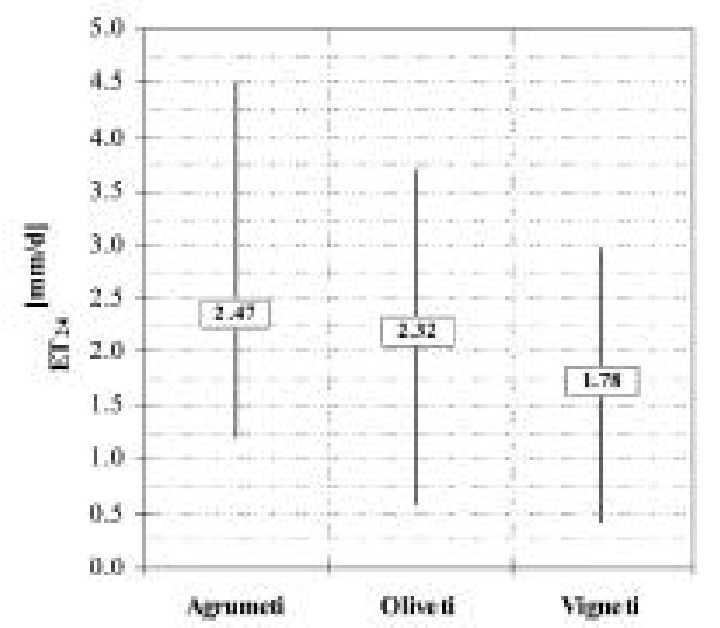

Fig. 8 - Stime di evapotraspirazione effettiva giornaliera per classi colturali.

Fig. 8 - Daily evapotranspiration values for different crops type. luzione geometriche adeguate alla caratterizzazione degli ambienti agricoli. In questo caso la possibilità di derivare a basso costo un elevato numero di osservazioni remote potrà rendere attuabile la metodologia proposta anche all'interno della pianificazione e gestione irrigua a scala comprensoriale.

\section{CONCLUSIONI}

\section{Conclusions}

L'indagine condotta ha dimostrato come l'impiego delle informazioni telerilevate da satellite e/o aereo possa oggi contribuire nella determinazione delle grandezze necessarie per l'applicazione, su vaste aree, di modelli agro-idrologici per la stima dei flussi di evapotra-spirazione. In particolare è stato preso in esame il modello SEBAL i cui algoritmi sono stati originariamente proposti per consentirne l'applicazione mediante dati telerilevati nelle bande VIS/NIR e TIR ed uno scarso numero di informazioni a terra.

Grazie ad una serie di dati sperimentali acquisiti in un'area test siciliana durante la stagione irrigua 2005, il modello SEBAL è stato inizialmente validato utilizzando misure di flussi energetici acquisiti con la tecnica scintillometrica.

Successivamente è stata verificata l'applicabilità del modello SEBAL a scala comprensoriale su un'area irrigua di circa 850 ettari in cui, nel 2002, è stato effettuato un volo aereo MIVIS ad alta risoluzione. La suddetta applicazione, nonostante alcuni limiti teorici del modello SEBAL che saranno approfonditi in successive applicazioni, ha consentito di stimare i tassi di evapotraspirazione effettiva giornaliera su tre tipologie di colture arboree, evidenziando la potenzialità operativa delle tecniche di osservazione terrestre a supporto della modellazione agro-idrologica.

\section{BIBLIOGRAFIA}

\section{References}

[1] Allen R.G., Pereira L.S., Raes D. e Smith M., Crop evapotranspiration. Guidelines for computing crop water requirements. FAO Irrigation and Drainage Paper n. 56 (1998), Rome, Italy.

[2] BASTIAANSSEN W.G.M., SEBAL-based sensible and latent heat fluxes in the irrigated Gediz Basin, Turkey, J. Hydrology (2000) 229: 87-100.

[3] BastiaAnssen W.G.M. e M.G. Bos, Irrigation performance indicators based on remotely sensed data: a review of literature, Irrigation and Drainage Systems (1999) 13: 291-311.

[4] Bastiaanssen W.G.M., Allen R.G., Droogers P., D'Urso G., Steduto P., Inserting man's irrigation and drainage wisdom into soil water flow models and bringing it back out: how far have we progressed, Workshop on "Unsaturated-Zone Modeling: Progress, Challenges and Applications", Wageningen University, The Netherlands 3-5 October 2004. 
[5] BastiaAnsSen W.G.M., HoEKMAn D.H. e RoEbeling R.A., A methodology for the assessment of surface resistance and soil water storage variability at mesoscale based on remote sensing measurements, IAHS Special Publications, (1994) No. 2, IAHS Press, Wallingford, Oxfordshire, UK

[6] Bastiaanssen W.G.M., Pelgrum H., Wang J., Ma Y., Moreno J., RoERINK G.J. e VAN DER WAL T., The Surface Energy Balance Algorithm for Land (SEBAL): Part 2 validation, J. of Hydr. (1998) 212-213: 213-229.

[7] Bastiannssen W.G.M., Menenti M., Feddes R.A. e Holtslag A.A.M., The Surface Energy Balance Algorithm for Land (SEBAL): Part 1 formulation, J. of Hydr. (1998) 212-213: 198-212.

[8] BRUTSAERT W., Evaporation into the atmosphere. Theroy, history and applications, D. Reidel Publ. Co. (1982), Dordrecht, 308 pp.

[9] BRUTSAERT W., Land-surface water vapor and sensible heat flux: Spatial variability, homogeneity, and measurement scales, Water Res. Research, (1998), vol. 34, pp. 2433-2442.

[10] BRUTSAERT W. e SUGITA M., Regional surface fluxes from satellite-derived surface temperatures (AVHRR) and radiosonde profiles, Bound. Layer Met., (1992), n. 58, pp. 355-366.

[11] Chodhury B.J., IDso S.B., Reginato R.J., Analysis of an empirical model for soil heat flux under a growing wheat crop for estimating evaporation by infrared-temperature based energy balance equation, Agric. and Forest Met., (1987), n. 39, pp. 283-297.

[12] CLEVERS J.G.P.W., The application of a weighted infrared-red vegetation index for estimating leaf area index by correcting for soil moisture, Remote Sens. Environm., (1989), 29.

[13] CRAgo R.D., Conservation and variability of the evaporative fraction during the daytime, J. of Hydr., (1996) 180, pp. 173-194.

[14] Criminisi A., Iovino M., Minacapilli M., Pumo D., Integrazione di un modello di simulazione idrologica in un Sistema Informativo Territoriale per la gestione dell'irrigazione in un comprensorio siciliano, AIIA 2001, Atti del convegno "Ingegneria Agraria per lo sviluppo dei paesi del mediterraneo" (2001) Vieste 11-14 settembre.

[15] D'Urso G., Simulation and Management of On-Demand Irrigation Systems: a combined agro-hydrological approach, PhD Dissertation (2001) Wageningen University, $174 \mathrm{pp}$

[16] Daughtry C.S.T., Kustas W.P., Moran M.S., PinTER P.J., JACKSON R.D., BRown P.W., Nichlos W.D. e GAY L.W., Spectral estimates of net radiation and soil heat flux, Rem. Sens. Env. (1990), n. 32, pp. 111124.

[17] De BruIN H.A.R., Introduction: Renaissance of Scintillometry, Boundary Layer Meteorol. (2002), 105, 1-4.

[18] ERDAS INC., ERDAS Field Guide. Fifth edition. (1999), Erdas Inc., Atlanta, USA.

[19] Hall F.G., Townshend J.R., Engman E.T., Status of Remote Sensing Algorithms for Estimation of Land Surface State Parameters, Remote Sens. Environm., (1995), 51: 138-156.

[20] Hillel D. (1998). Environmental soil physics. Academic Press, London, UK, 771 pp.

[21] Kustas W.P., Norman J.M., ANDERSON M.C., FrenCH A.N. (2003). Estimating subpixel surface temperatu- res and energy fluxes from the vegetation index-radiometric temperature relationship. Remote Sens. Environ. 85, 429-440.

[22] LI-CoR, LAI-2000 plant canopy analyzer operating manual, Li-Cor: Lincoln, NE. 1992.

[23] Minacapilli M., D’uRso G., QIANG L., Applicazione e confronto dei modelli SAIL e CLAIR per la stima dell'indice di area fogliare da dati iperspettrali MIVIS, Rivista Italiana di Telerilevamento, (2005), 33/34, 15-25.

[24] Meijninger W.M.L., Surface fluxes over natural landscapes using scintillometry, $\mathrm{PhD}$ Dissertation, (2003) Wageningen University, 176 pp.

[25] Menenti M., Physical aspects and determination of evaporation in desert applying remote sensing techniques, ICW report n.10 (Special issue) (1984).

[26] Menenti M., Evaporation, Remote Sensing in Hydrology and Water Management. Springer-Verlag Inc., (2000), G.A. Schultz and E.T. Engman (eds.), New York, U.S.A., pp. 155-189.

[27] Menenti M., Choudhury B.J., Parameterization of land surface evaporation by means of location dependent potential evaporation and surface temperature range, In: Exchange Processes at the Land Surface for a Range of Space and Time Scales. Bolle, H.J., et al. (eds.), IAHS Publ. (1993), n. 212, pp. 561-568.

[28] Menenti M., Visser T.N.M., Morábito J.A., DroVANDI A., Appraisal of irrigation performance with satellite data and georeferenced information, In: The impact of satellite sensor resolution on the regional evaporation statistics in large-scale irrigation schemes, Roerink G.J., (1994), DLO Winand Staring Centre-Wageningen Agricultural University, Wageningen, The Netherlands, pp. 6-7.

[29] Monteith J.L. e Unsworth M.H., Principles of environmental physics, (1990), E. Arnold, London, 290 pp.

[30] Morse A., Tasumi M., Allen R.G., Kramber W., Application of the SEBAL Methodology for Estimating Consumptive Use of Water and Streamflow Depletion in the Bear River Basin of Idaho through Remote Sensing, Final report submitted to the Raytheon Systems Company, Earth Observation System Data and Information System Project, by Idaho Department of Water Resources and University of Idaho, (2000) 107 pages.

[31] PAULSON C.A., The mathematical representation of windspeed and temperature profiles in unstable atmospheric surface layer, J. Appl. Meteorol. (1970) 9 : 857-861.

[32] Slater P., Biggar S., Thome K., Gellman D. e SPYAK P., Vicarious radiometric calibrations of EOS sensors, Journal of Atmospheric and Ocean Technology, (1996), 13: 349-359.

\section{Parole chiave:}

Evapotraspirazione; Telerilevamento; SEBAL;

\section{SUMMARY}

\section{EVAPOTRANSPIRATION ASSESSMENT BY MEANS OF HYPERSPECTRAL AIR-BORNE REMOTE SENSING DATA}

The general aim of this paper is to demonstrate that remote sensing techniques may easily provide the information needed for the application, over large areas, 
of agro-hydrological models for the actual evapotranspiration estimation.

The application of the SEBAL model has been investigated at field scale and at district scale using highresolution hyperspectral (VIS/NIR + TIR) airborne data acquired in a Sicilian test area. In a first phase, SEBAL has been validated by means of scintillometer flux measurements. Then an application over a district area using MIVIS airborne data has been performed.
The procedure provides the actual daily evapotranspiration distribution for a set of irrigated arboreous crops, confirming the potential advantage of this approach as an operative future tool useful to improve the efficiency of irrigation systems.

\section{Key words:}

Evapotranspiration; Remote Sensing; SEBAL. 